\title{
Iterative reconstruction of thermally induced phase distortion in a $\mathrm{Nd}^{3+}: \mathrm{YVO}_{4}$ laser
}

Ludovic Grossard, ${ }^{*}$ Agnès Desfarges-Berthelemot, Bernard Colombeau, and Claude Froehly Institut de Recherche en Communications Optiques et Microondes

123, av. Albert Thomas, 87060 Limoges Cedex, France

\begin{abstract}
The thermal distortions in a CW end-pumped $\mathrm{Nd}: \mathrm{YVO}_{4}$ laser are experimentally investigated by use of an external $\mathrm{TEM}_{00}$ probe beam. After a round-trip of this probe through the active medium, three irradiance profiles are recorded along its propagation axis. The distorted phase pattern of this probe beam is then reconstructed from these profiles by a Gerchberg-Saxton-like iterative algorithm. To point out the contribution of each kind of aberration in the phase profile, we have computed a Zernike polynomial expansion, and have shown that the main terms constituting the aberration are (in order) defocusing (optical path difference (OPD) $0.7 \lambda$ FWHM), spherical aberration (OPD $0.3 \lambda$ FWHM) and first order astigmatism (OPD of $\pm 0.14 \lambda_{p}$ FWHM). Moreover, all higher order terms result in a significant phase peak $(\lambda / 4 \mathrm{FWHM}$ over $400 \mu \mathrm{m})$.

PACS numbers:
\end{abstract}

Keywords:

*Electronic address: grossard@ircom.unilim.fr 


\section{INTRODUCTION}

During the last few years, end-pumped solid-state lasers have expanded rapidly partly because of their high conversion efficiency, easy transverse mode selection due to gain distribution inside the amplifying medium, and much appreciated ease of use. Moreover, the good spatial overlap between the pump and laser beams on one hand, and the spectral matching between the pump beam wavelength and laser crystal absorption bandwidth on the other hand, have strongly reduced thermal effects in the active medium. However, with higher diode power becoming recently available, thermal effects have become again a serious limitation of the laser brightness, leading to either a multimodal emission or severe power losses if a $\mathrm{TEM}_{00}$ laser beam is maintained by intracavity filtering.

Wavefront aberrations can be corrected in real-time using a phase conjugation technique by four-wave mixing for example $[1,2]$. However, this process induces a dramatic global efficiency fall because of high power pump beam requirement. If the thermal distortions are time-independent, the phase compensation can be performed in a static way. The thermal aberration reduction can be obtained for example by longitudinally cooling the amplifying medium [3], by designing a resonator geometry in which the laser beam size in the active medium is much smaller than the pump beam size [4], but the corresponding efficiency is low. The phase correction can also be reached with a well-suited rectifying phase plate inserted in the cavity [5]. In the latter case, the thermal aberration could be entirely corrected if it is fully characterized.

Such a characterization has been performed for a CW end-pumped Nd:YAG laser thanks to an interferometric device [4].

In this paper, we present the thermal effect determination in an end-pumped CW Nd:YVO 4 laser with a non-interferometric method where the distorted phase profiles are calculated thanks to an iterative reconstruction process using irradiance records. Such a technique has been previously used in stellar imaging to determine the Hubble Space Telescope wave-front distortions [6], and we have, for the first time to our knowledge, applied this method to measure the thermal effects in a running laser.

Firstly, we show experimentally that high pumping power leads to a spatially multimodal regime without any intracavity spatial filtering, pointing out the need of an external $\mathrm{TEM}_{00}$ probe beam. Then, we present the probe beam distortion measurements when the pumping power is equal to $9 \mathrm{~W}$. After a round-trip in the amplifying medium, irradiance profiles of this probe beam are recorded in several planes along its propagation axis. At least two planes are required [7] to reconstruct 
the optical field from irradiance measurements. However, using only two Fourier transform planes, we did not recovered the measured irradiance patterns from the reconstructed wavefront. That is why we used the irradiance profile in a third plane. Experimentally, the considered planes were the image of the active medium output plane, its Fourier transform plane and a Fresnel one. The distorted wavefront is then reconstructed by an iterative Gerchberg-Saxton-like algorithm [7] using the three preceeding irradiance measurements. Finally, the calculated phase profile in the laser crystal output plane is analyzed using a Zernike polynomial expansion and main aberration terms are pointed out.

\section{WAVEFRONT DISTORTION MEASUREMENT METHOD}

\section{A. Laser wavefront distortion and beam divergence}

The laser studied here is represented in figure 1 . A $3 \mathrm{~mm}$ thick $1.1 \% \mathrm{Nd}^{3+}: \mathrm{YVO}_{4}$ crystal is longitudinally end-pumped by a CW pigtailed laser diode at $808 \mathrm{~nm}$. The active medium is birefringent and the laser beam is linearly polarized along the extraordinary axis. The mirror $\mathrm{M}_{1}$ is totally reflecting for the laser line and anti-reflection coated at $808 \mathrm{~nm}$. The power reflectance of the second mirror $\mathrm{M}_{2}$ is equal to $95 \%$. The two mirrors are located in the focal planes of a converging lens $\mathrm{L}_{1}$ whose focal length is equal to $400 \mathrm{~mm}$. This resonator is known as a FourierTransform cavity.

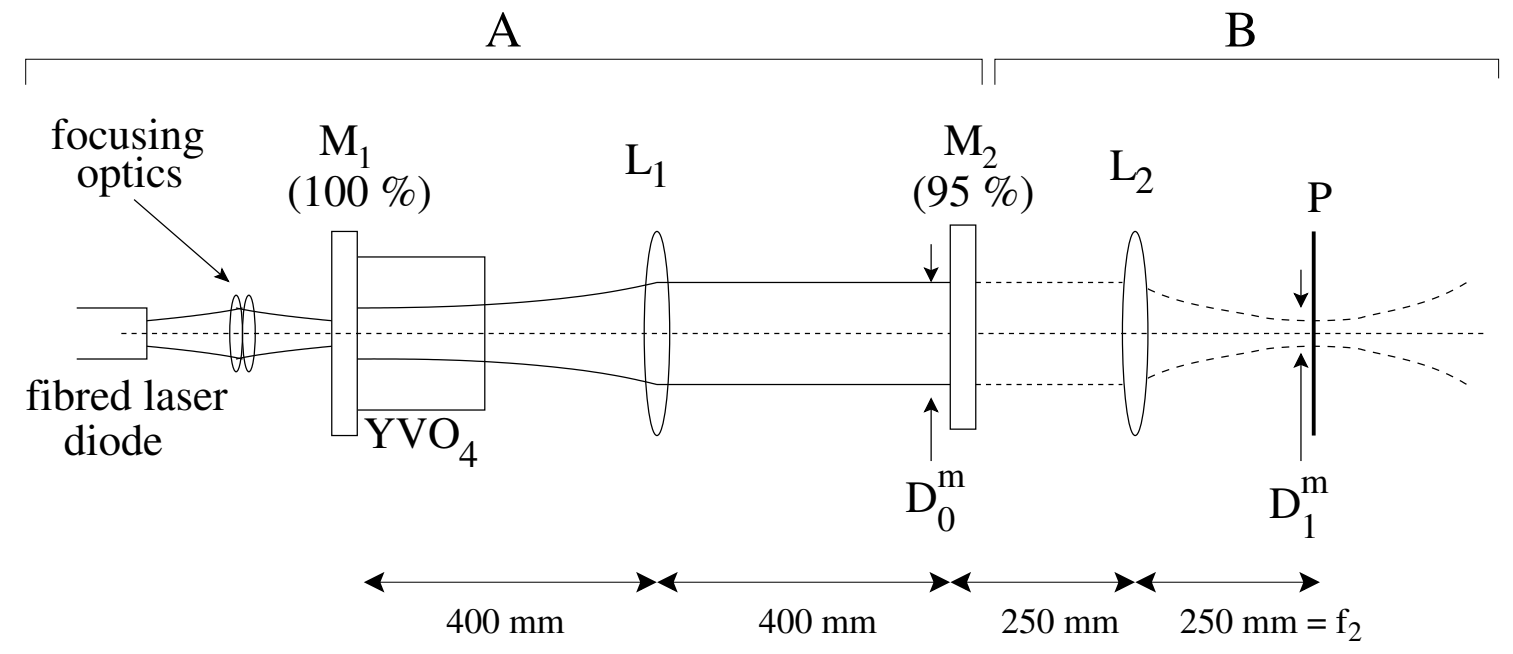

FIG. 1: A: Laser studied for the characterization of thermal effects. B: divergence parameter measurement.

Near the threshold, a $\mathrm{TEM}_{00}$ beam is emitted due to tri-dimensional gain filtering in the laser medium without additional spatial filtering by any aperture. If the pumping power is increased, 
the energy deposited in the laser crystal leads to a well-known thermal lens effect. Without any precaution, the laser mode fits to the aberrating medium so that the wavefronts remain plane in the mirror planes. This leads to an increased beam divergence. It can be estimated from the measurements of the laser beam width (FWHM) $D_{0}^{m}$ in the plane of mirror $M_{2}$ and the laser beam width (FWHM) $D_{1}^{m}$ in the focal plane $\mathrm{P}$ of the lens $\mathrm{L}_{2}\left(\mathrm{f}_{2}=250 \mathrm{~mm}\right)$. With a $\mathrm{TEM}_{00}$ beam having a diameter equal to $D_{0}^{m}$ in the plane of $\mathrm{M}_{2}$, the calculated beam diameter $D_{1}^{c}$ is equal to $2(\ln 2) \lambda f_{2} / \pi D_{0}^{m}$ in the $\mathrm{P}$ Plane. We defined the ratio $k=D_{1}^{m} / D_{1}^{c}$ as a divergence measurement.

We observed (figure 2) that the divergence parameter $\mathrm{k}$ quickly increases from 1 near the threshold to about 18 at $9.7 \mathrm{~W}$ pumping. This means that near the threshold, the laser beam can be considered as diffraction limited, whereas under strong pumping power, the beam size $D_{1}^{m}$ is 18 times what it should be if the beam profile was monomode. Under pumping power equal to $9.7 \mathrm{~W}$, thermal lens effects do not result in an efficiency fall, but in a deterioration of the laser beam spatial quality, leading to a strong decrease in brightness. A $\mathrm{TEM}_{00}$ beam profile can be maintained by placing a spatial filter in the resonator, but the laser efficiency decreases because of important losses due to filtering.

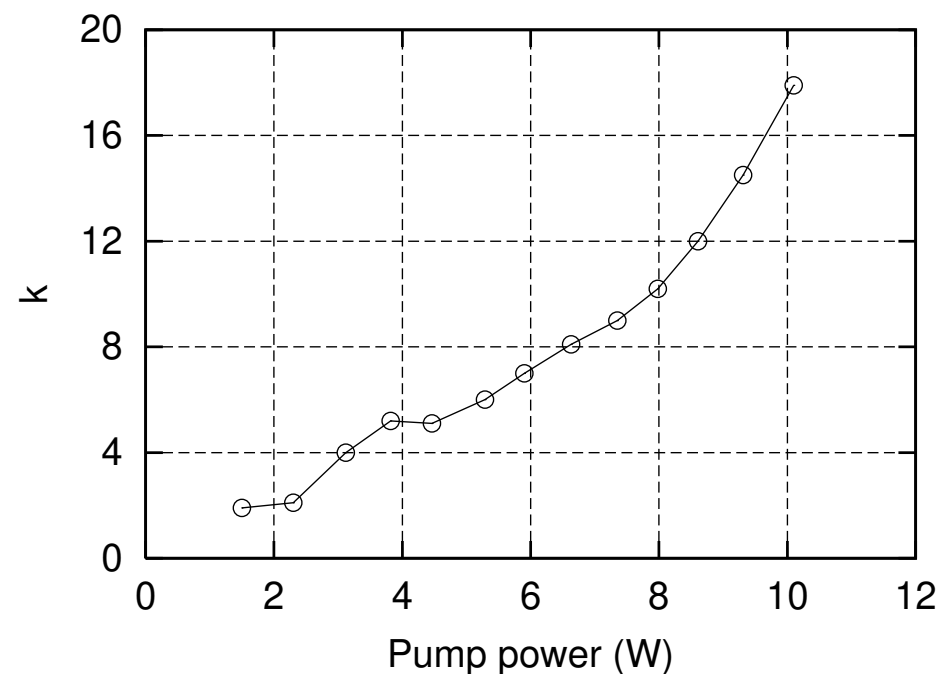

FIG. 2: Divergence parameter of the laser beam versus the pumping power: $k=\frac{\pi D_{0}^{m} D_{1}^{m}}{2(\ln 2) \lambda f_{2}}$.

\section{B. External probe of wavefront distortions}

When the pumping power is increased, the phase pattern of the laser beam is complicated because of the multimodal emission. This modal structure arises from the thermal distortion 
effects on one hand, and from the plane wavefront requirement onto the resonator mirrors on the other hand. It is therefore impossible to determine the thermally induced index pattern in the amplifying medium analyzing the laser beam itself. A solution consists in using an external probe plane wave and analyzing its wavefront distortions after a round-trip in the amplifying medium. The distorted phase profile of the probe wave can be classically determined by interferometry. Here, we have developed an alternative method where this profile is calculated thanks to three irradiance profile records of the probe beam using a Gerchberg-Saxton-like phase retrieval algorithm.

\section{EXPERIMENTAL SETUP}

The experimental setup is shown in figure 3. This arrangement is divided into three parts: the probe YAP laser, the $\mathrm{YVO}_{4}$ laser and the irradiance measurement device.

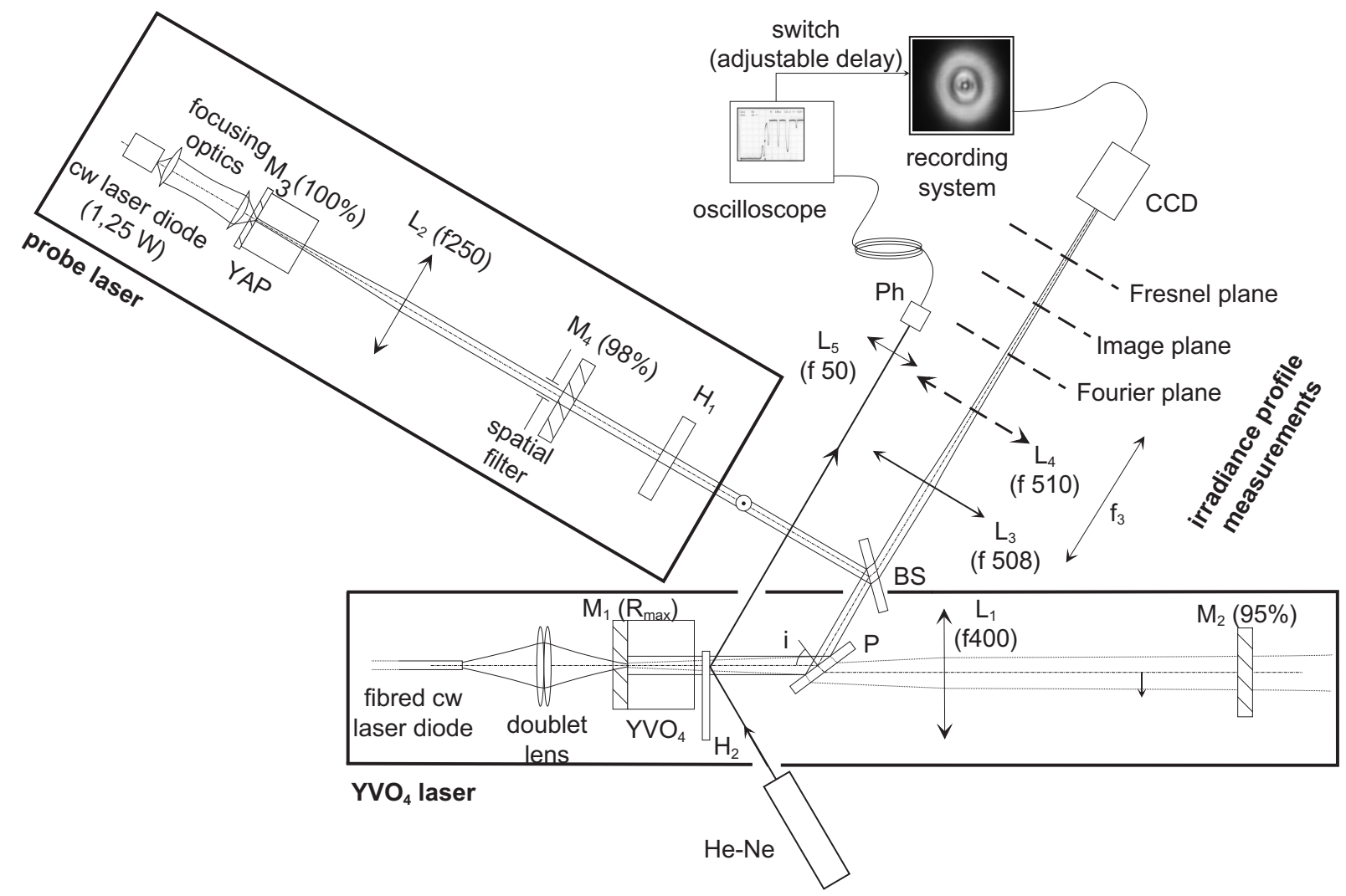

FIG. 3: Experimental setup used for the characterization of the thermal effects in an end-pumped $\mathrm{YVO}_{4}$ laser. $\mathrm{M}_{1}, \mathrm{M}_{2}, \mathrm{M}_{3}, \mathrm{M}_{4}$ : mirrors; $\mathrm{L}_{1}, \mathrm{~L}_{2}, \mathrm{~L}_{3}, \mathrm{~L}_{4}, \mathrm{~L}_{5}$ : lenses; $\mathrm{P}$ polarizing plate; $\mathrm{BS}$ beam splitter; $\mathrm{Ph}$ : photodiode; $\mathrm{H}_{1}, \mathrm{H}_{2}$ : half wave plate. The image plane is obtained with the lenses $\mathrm{L}_{3}$ and $\mathrm{L}_{4}$ whereas the Fourier and Fresnel planes are obtained with the lens $\mathrm{L}_{3}$ only. 


\section{A. Probe beam characteristics}

The cavity of the probe laser is a Fourier-transform one to obtain a laser beam with a large stability length. The active medium is a YAP: $\mathrm{Nd}^{3+}$ crystal pumped by a CW laser diode at $808 \mathrm{~nm}$. The mirrors $M_{3}$ and $M_{4}$ are located in the focal planes of the lens $L_{2}$ (focal length $250 \mathrm{~mm}$ ). A spatial filter is placed in the plane of mirror $\mathrm{M}_{4}$ to obtain a high spatial quality $\mathrm{TEM}_{00}$ output laser beam. The probe beam wavelength is chosen equal to $1079 \mathrm{~nm}$, i.e. outside the gain bandwidth of the $\mathrm{YVO}_{4}$ crystal, to avoid modifications of the population inversion. However, this wavelength is close enough to the $\mathrm{YVO}_{4}$ emission wavelength to limit chromatic dispersion effects. The probe beam is linearly polarized, and its direction of polarization is adjusted by a half-wave plate $\mathrm{H}_{1}$ located outside the resonator.

The probe beam size near mirror $\mathrm{M}_{4}$ is equal to $1 \mathrm{~mm}$ (FWHM), corresponding to a Rayleigh length about $3 \mathrm{~m}$. The distance between the mirror $\mathrm{M}_{4}$ and the $\mathrm{YVO}_{4}$ crystal being approximately equal to $1.5 \mathrm{~m}$, the field curvature of the probe beam in the crystal is not significant. This ensures that the $\mathrm{YVO}_{4}$ crystal is probed by a quasi plane-limited wavefront and that the reconstructed phase profile will not be affected by the intrinsic curvature of the probe beam.

\section{B. polarization requirements}

The index change versus temperature in the $\mathrm{YVO}_{4}$ crystal is different along the ordinary and extraordinary axis, and therefore the probe beam and laser beam must have the same polarization in the laser medium. Under this condition, the probe beam injection leads to dramatic power losses for the $\mathrm{YVO}_{4}$ laser. To overcome this problem, the laser emission is stopped when the amplifying medium is probed. The measurements are synchronized with the laser shutdown to avoid crystal warm up because of non radiative transitions. The probe beam injection is performed thanks to the polarizing plate $\mathrm{P}$ inserted into the cavity. This plate is at Brewster incidence with the laser axis and is totally reflecting for the vertical polarization and AR coated for the horizontal one. The probe beam is then horizontally polarized by rotating the half wave plate $\mathrm{H}_{1}$ and the laser beam is horizontally polarized.

For the laser shutdown and measurement triggering off, we used the following arrangement: firstly, the laser is running and thermal stability is achieved. The probe beam is directed into the vanadate crystal with vertical polarization (figure 4.a). Secondly, a half wave $H_{2}$ plate is inserted between the polarizing plate $\mathrm{P}$ and the $\mathrm{YVO}_{4}$ crystal. The trace of the polarization plane on the 
$\mathrm{H}_{2}$ plate surface bisects the angle between the axes of the plate, so that the probe beam undergoes a $90^{\circ}$ rotation and does a round-trip in the laser crystal with the same polarization as the laser beam. The laser does not work anymore because of losses added by the set "polarizing plate $\mathrm{P}+$ half-wave plate $\mathrm{H}_{2} "$ (figure 4.b).

a)

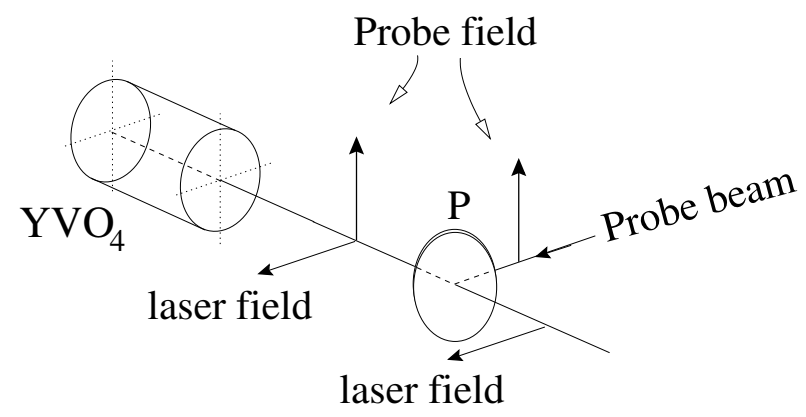

b)

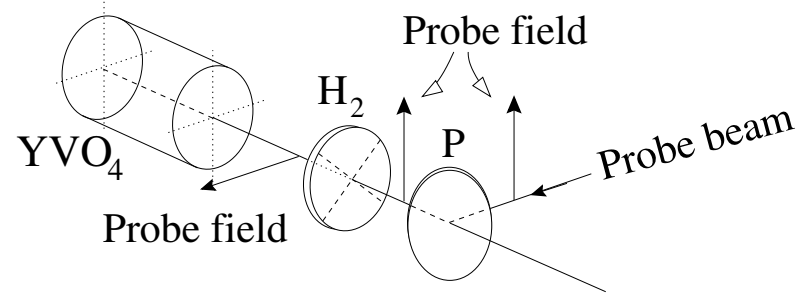

FIG. 4: Probe beam injection method. P: polarizing plate, $H_{2}$ : half wave plate. a): the laser is running and the probe field is vertically polarized. b): $H_{2}$ is inserted into the resonator. The laser emission is shutdown and the probe field is horizontally polarized. The measurements are performed as soon as possible to avoid crystal warmup.

\section{C. measurement triggering off}

The synchronization is obtained by use of an He-Ne laser beam and a photodiode as shown in figure 3. This beam is focused on the photodiode $\mathrm{Ph}$ thanks to a $50 \mathrm{~mm}$ focal length lens $L_{5}$. The half-wave plate $\mathrm{H}_{2}$ is inserted into the laser thanks to an electric motor and crosses the $\mathrm{YVO}_{4}$ laser beam for about about $1 \mathrm{~ms}$. We used the reflection of the He-Ne laser beam onto this plate to start the measurements. The voltage given by the resistor associated with the photodiode is used to trigger off an oscilloscope. A second signal is generated by the oscilloscope to activate the recording system, allowing us to add an adjustable delay.

\section{D. description of the irradiance measurement device}

After a round-trip in the laser medium, the probe beam is partly transmitted through the beam splitter BS, and defines the axis along which measurements of three irradiance patterns are performed: 
- The first record is that of the image of the laser crystal output plane. This is obtained thanks to the couple of lenses $\mathrm{L}_{3}$ and $\mathrm{L}_{4}$, whose focal lengths are respectively equal to $508 \mathrm{~mm}$ and $510 \mathrm{~mm}$. These lenses are positioned so that the laser crystal output plane is located in the object plane of $\mathrm{L}_{3}$, and a CCD camera is placed in the Fourier plane of $\mathrm{L}_{4}$. The distance between the two lenses is equal to $116 \mathrm{~mm}$.

- Secondly, we record the irradiance of a field pattern which is a Fourier transform of the previous one. We simply removed $\mathrm{L}_{4}$ and placed the camera in the Fourier plane of $\mathrm{L}_{3}$.

- The third irradiance pattern is recorded in a Fresnel plane obtained by moving the CCD camera away from the Fourier plane by $270 \mathrm{~mm}$.

Irradiance patterns are recorded with a TM-745 CCD camera (Pulnix) whose pixel size is equal to $11 \mu \mathrm{m} \times 11 \mu \mathrm{m}$. The irradiance recorded by each pixel is coded with 256 grey levels.

\section{EXPERIMENTAL RESULTS}

\section{A. irradiance profiles}

Irradiance profiles were recorded in the three previously defined planes (named hereafter: image, Fourier and Fresnel planes). These measurements were performed using three different experimental configurations (figure 5):

- The laser crystal is not pumped. This allows us to verify that the intrinsic phase curvature of the probe beam (i.e only due to propagation) in the $\mathrm{YVO}_{4}$ crystal is negligible.

- The pumping power is equal to $9.3 \mathrm{~W}$. The irradiance profiles are recorded only some milliseconds after laser shutdown. These records will be further used to effectively determine the distorted phase profile.

- The pumping power is equal to $9.3 \mathrm{~W}$. The irradiance profiles are recorded several seconds after laser shutdown in order to point out the influence of the crystal warmup when the pumping power is no longer removed by laser emission.

For each record, the probe beam power is adjusted to optimize the record dynamic. This explains that the profiles recorded several seconds after the laser shutdown seem brighter than 

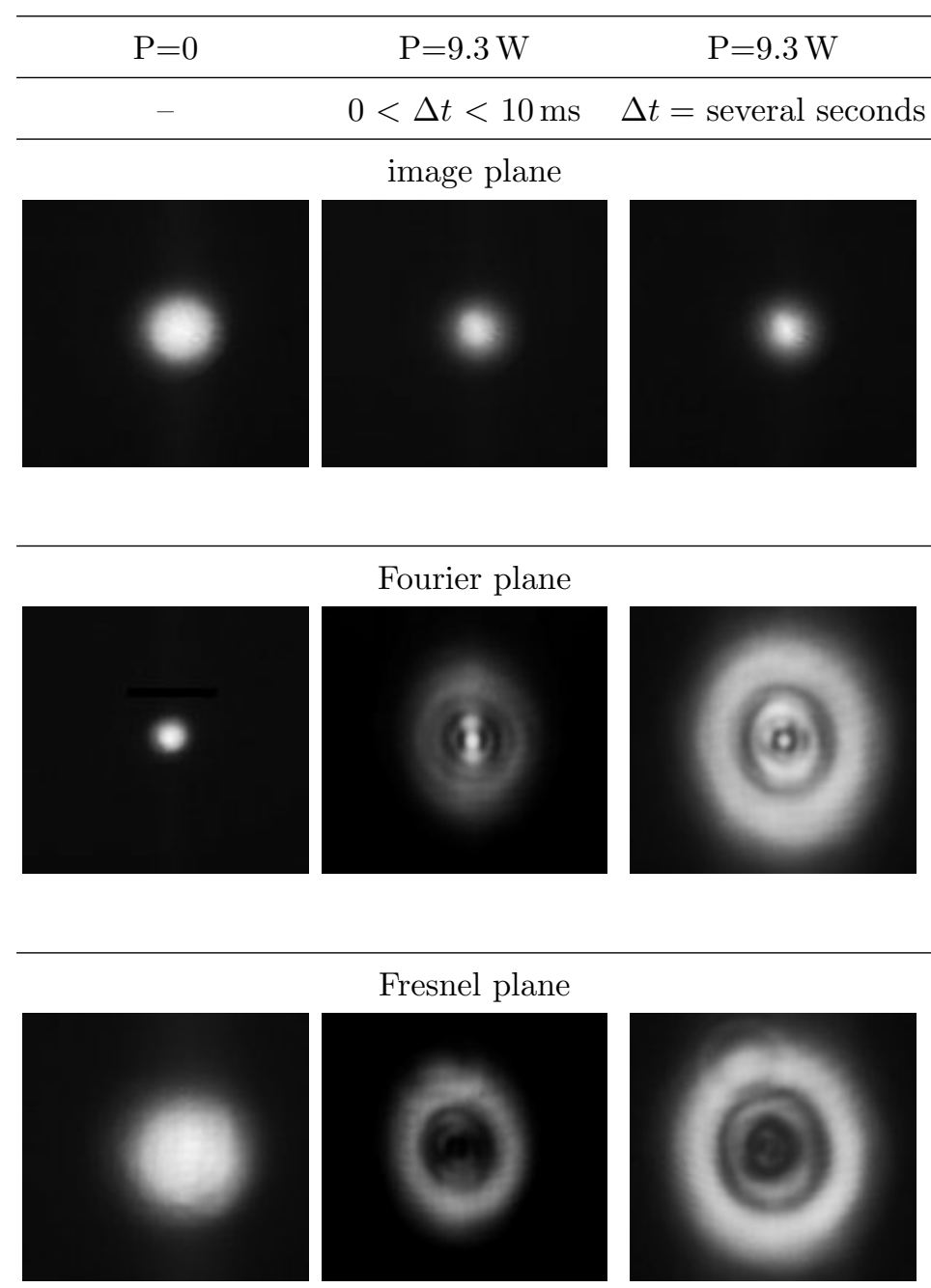

FIG. 5: Measured irradiance profiles in the image, Fourier and Fresnel planes. Column 1: pumping power $\mathrm{P}=0 \mathrm{~W}$. Column 2: $\mathrm{P}=9.3 \mathrm{~W}$ and profiles recorded $10 \mathrm{~ms}$ after the laser shutdown. Column $3: \mathrm{P}=9.3 \mathrm{~W}$ and profiles recorded several seconds after the laser shutdown.

those recorded only some milliseconds later: in fact, the whole energy is the same for all profiles, but the distribution is not the same for each of them.

The elliptically observed irradiance distribution in the Fourier plane could be attributed to temperature gradient anisotropy and thermal stress in the amplifying medium, leading to an anisotropic index pattern. 


\section{B. phase retrieval algorithm}

The phase profile of the probe beam was determined using a Gerchberg-Saxton like algorithm. This method allows us to reconstruct the distorted wavefront from a set of irradiance records along its propagation axis. A simplified version of the developed algorithm is shown in figure 6 . Let us briefly discuss the various steps of this cyclic algorithm

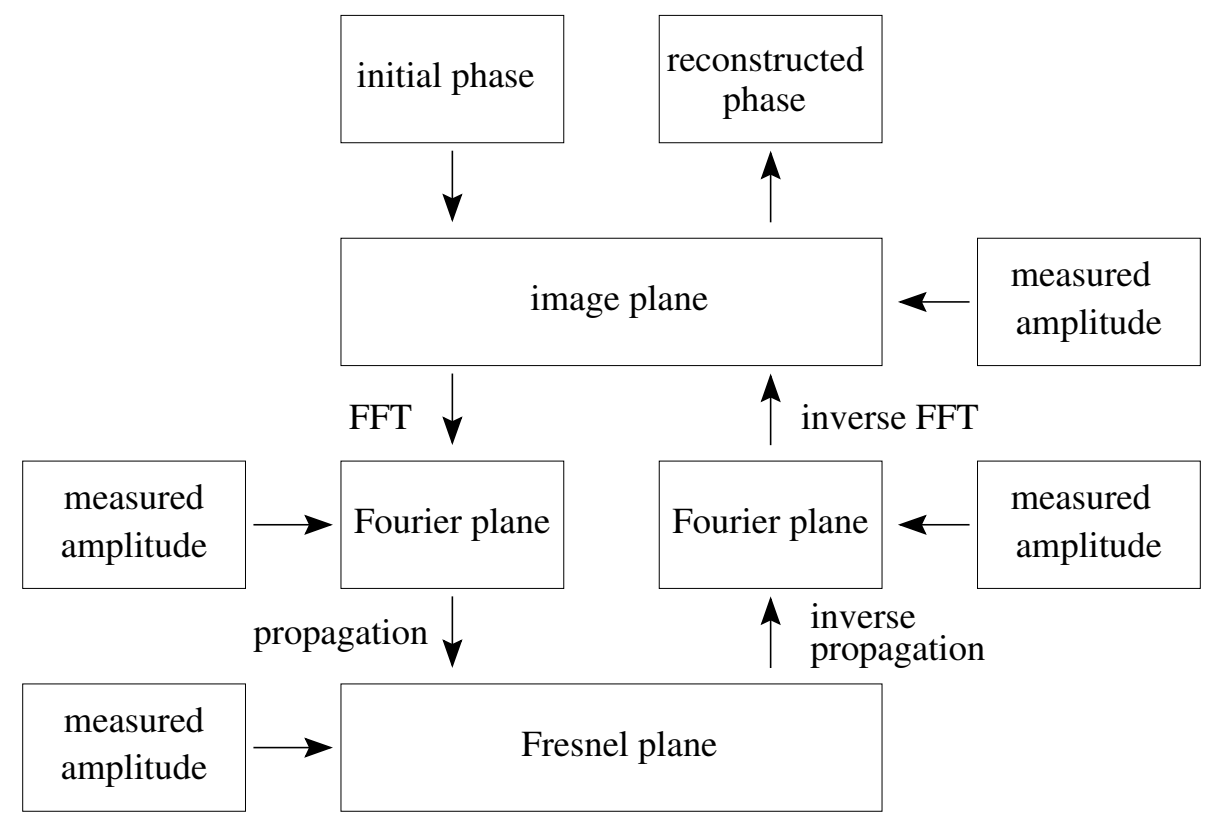

FIG. 6: Numerical algorithm derived from Gerchberg-Saxton algorithm and used for phase retrieval of the probe beam. The experimental data are amplitude profiles measured in three successive planes.

To begin, the phase profile in the image plane is set equal to zero as a first guess. The complex field in this plane is then constructed by multiplying this initial phase with the measured amplitude (which is proportional to the square root of the measured irradiance). This field undergoes a Fourier-transform to give the complex field in the Fourier plane. The calculated amplitude is then removed and replaced by the measured one, while keeping the calculated phase profile unchanged. The complex field in the Fresnel plane is computed using the beam propagation method. Again, the calculated amplitude is replaced by the measured amplitude, while maintaining the calculated phase profile. This new complex field is then propagated back to the Fourier plane where the calculated amplitude is replaced again. An inverse Fourier transform operation gives the optic field in the initial plane, and so on...

The whole process is iterated until the calculated phase profile converges. After each iteration, the current error is estimated from the measured amplitude and from the reconstructed one in the 
Fourier plane. This error is calculated using the following expression:

$$
\epsilon=\sqrt{\frac{\sum_{i, j}\left(I_{2}-I_{2}^{r}\right)^{2}}{\sum_{i, j}\left(I_{2}\right)^{2}}}
$$

where $I_{2}$ and $I_{2}^{r}$ denote the measured and reconstructed irradiance profiles in the Fourier plane. $i$ and $j$ are the coordinates of each pixel of the images. The convergence criterion is given by the current error stagnation.

Firstly, this algorithm was used to determine the intrinsic phase curvature of the probe beam in the laser crystal without thermal effects. Using the irradiance profiles obtained without pump beam, we found an intrinsic phase curvature of the probe beam corresponding to an optical path difference (OPD) of $0.07 \lambda_{p}(\mathrm{FWHM})$ where $\lambda_{p}$ denotes the probe wavelength. The convergence of the algorithm was reached after only 20 iterations. Thus, this wavefront can be considered as a plane wave, and phase distortions of the probe beam under strong pumping conditions are directly linked to thermal aberrations.

Secondly, the probe beam phase profile was reconstructed thanks to irradiance profiles recorded while pumping the laser medium, $10 \mathrm{~ms}$ after the laser emission shutdown.

The reconstructed and measured irradiance profiles in the three working planes are shown in figure 7. The reconstructed ones were obtained after 63 iterations. Column 1 represents reconstructed profiles using a constant value (plane wavefront) as initial phase in the image plane. To emphasize that the reconstructed beam profiles do not depend on initial condition, we performed again the cyclic computation with the same initial phase, but in the Fourier plane (column 2). For comparison, the measured profiles are shown in column 3.

The reconstructed phase profile in the image plane and the corresponding irradiance profile are shown in figure 8. Only phase values corresponding to irradiances much greater than recording noise should be taken into account.

To entirely characterize the thermal aberration, we propose to estimate the contribution of each kind of well-known aberrations thanks to a Zernike polynomial expansion of the reconstructed phase profile [8].

\section{Zernike polynomial expansion of the reconstructed phase profile}

The well-known Zernike polynomials are related to the classical aberrations and thus provide a convenient mathematical expression of the aberration content in a wavefront using familiar terms. 


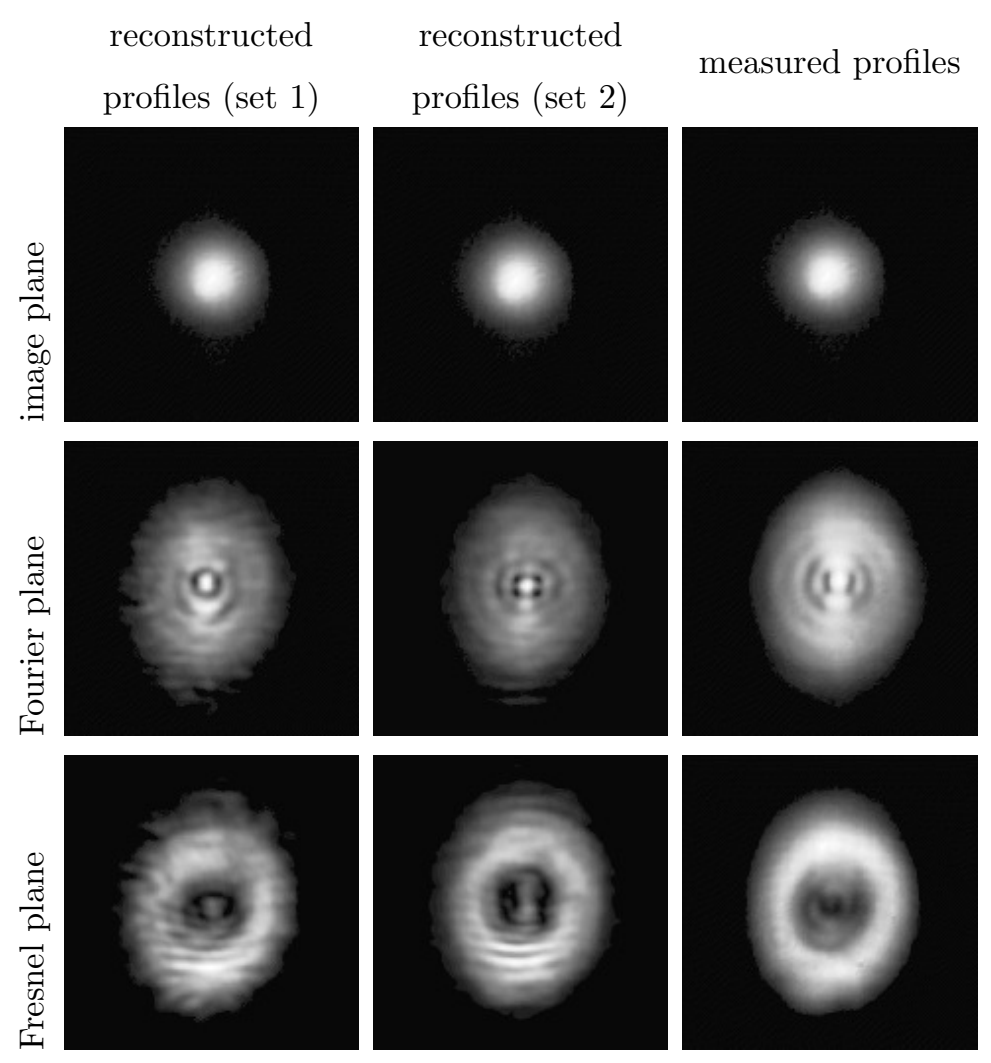

FIG. 7: Reconstructed and measured irradiance profiles obtained with our algorithm in the image, Fourier and Fresnel planes. Set 1: plane wavefront as initial condition in the image plane, set 2: plane wavefront as initial condition in the Fourier plane.

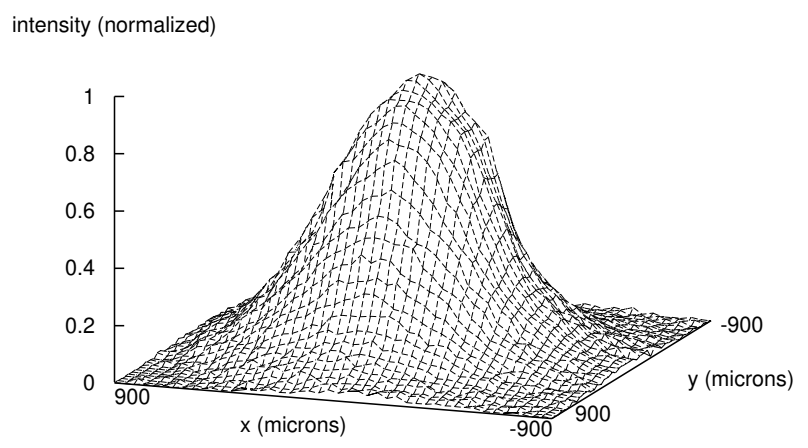

a) measured irradiance profile

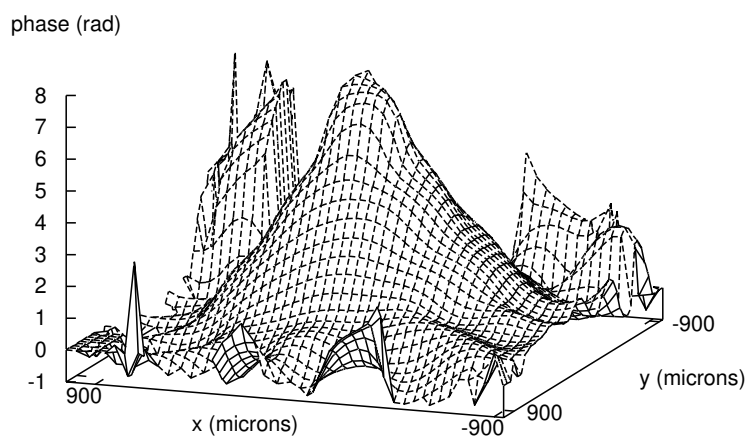

b) reconstructed phase profile

FIG. 8: measured irradiance profile and reconstructed phase profile in the image plane under strong thermal effects. 
The Zernike polynomial expansion of the phase profile $\phi(\rho, \theta)$ is given by:

$$
\phi_{1}(\rho, \theta)=\sum_{n=0}^{k} \sum_{m=0}^{n} A_{n m} R_{m}^{n-2 m}(\rho) \begin{aligned}
& \sin (n-2 m) \theta \\
& \cos (n-2 m) \theta
\end{aligned}
$$

where the sinus (resp. cosines) function is used when $n-2 m>0$ (resp. $n-2 m \leqslant 0$ ). $A_{n m}$ are the coefficients of the expansion and $R_{m}^{n-2 m}(\rho)$ denotes the Zernike polynomial. $\rho$ and $\theta$ correspond to the polar coordinates.

Table I represents the first fifteen Zernike coefficients with the corresponding aberrations.

\begin{tabular}{|c|c|c|c|c|c|}
\hline num & $\mathrm{n}$ & $\mathrm{m}$ & polynomial & Definition & $A_{n m}$ \\
\hline 1 & 0 & 0 & 1 & piston & 2.2694 \\
\hline 2 & 1 & 0 & $r \sin \theta$ & tilt $Y$ & -0.0158 \\
\hline 3 & 1 & 1 & $r \cos \theta$ & tilt $\mathrm{X}$ & -0.1601 \\
\hline 4 & 2 & 0 & $r^{2} \sin (2 \theta)$ & 1st order astigmatism $45^{\circ}$ & -0.2425 \\
\hline 5 & 2 & 1 & $2 r^{2}-1$ & defocusing & -2.3337 \\
\hline 6 & 2 & 2 & $r^{2} \cos (2 \theta)$ & 1st order astigmatism $0^{\circ}$ & 0.8494 \\
\hline 7 & 3 & 0 & $r^{3} \sin (3 \theta)$ & trefoil $30^{\circ}$ & -0.0174 \\
\hline 8 & 3 & 1 & $\left(3 r^{3}-2 r\right) \sin (\theta)$ & coma $\mathrm{Y}$ & -0.0127 \\
\hline 9 & 3 & 2 & $\left(3 r^{3}-2 r\right) \cos (\theta)$ & coma X & 0.1772 \\
\hline 10 & 3 & 3 & $r^{3} \cos (3 \theta)$ & trefoil $0^{\circ}$ & -0.0452 \\
\hline 11 & 4 & 0 & $r^{4} \sin (4 \theta)$ & tetrafoil $22,5^{\circ}$ & -0.0361 \\
\hline 12 & 4 & 1 & $\left(4 r^{4}-3 r^{2}\right) \sin (2 \theta)$ & 2 nd order astigmatism $45^{\circ}$ & -0.1538 \\
\hline 13 & 4 & 2 & $6 r^{4}-2 r^{2}-1$ & spherical aberration & 1.2419 \\
\hline 14 & 4 & 3 & $\left(4 r^{4}-3 r^{2}\right) \cos (2 \theta)$ & 2nd order astigmatism $0^{\circ}$ & -0.3399 \\
\hline 15 & 4 & 4 & $r^{4} \cos (4 \theta)$ & tetrafoil $0^{\circ}$ & -0.0829 \\
\hline
\end{tabular}

TABLE I: Zernike polynomial expansion coefficients for the reconstructed phase profile in the image plane.

Three kinds of well-know aberrations were found to be significant:

- Defocusing (OPD of $0.7 \lambda_{p}$ FWHM).

- Spherical aberration (OPD of $0.3 \lambda_{p}$ FWHM).

- First order astigmatism (OPD of $\pm 0.14 \lambda_{p}$ (FWHM), the sign depending on the axis).

- Higher order aberration: $\lambda_{p} / 4$ curvature on $400 \mu m$. 


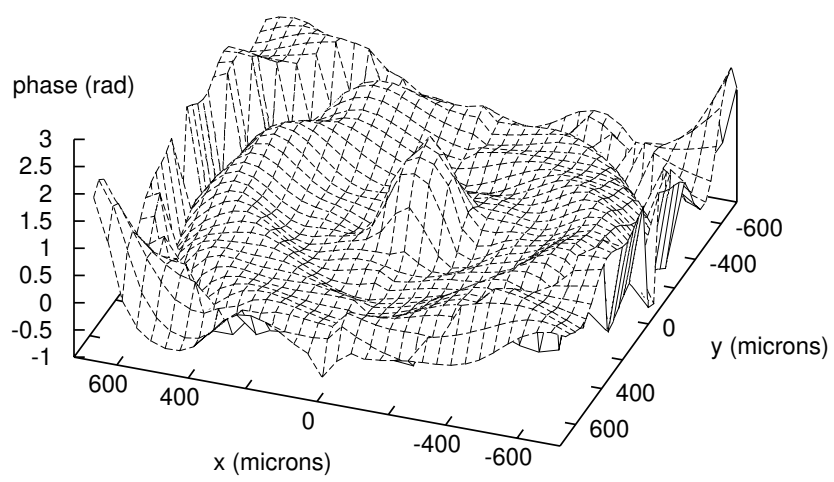

FIG. 9: Phase distortion after defocusing, spherical aberration and first order astigmatism removal. This residual aberration is characterized by a $\lambda_{p} / 4$ OPD (FWHM) over a $400 \mu \mathrm{m}$ diameter.

The distorted phase profile is mainly constituted by the parabolic term, due to the non-aberrant thermal lens. This phase contribution has no practical importance for further aberration compensation as it can be canceled by simply translating the internal lens of the resonator.

It appears that spherical aberration and first order astigmatism cannot be neglected compared to the parabolic term and should be taken into account to obtain an efficient phase correction. Moreover, the sum of all higher order aberrations result in a significant phase curvature (OPD $\lambda_{p} / 4 \mathrm{FWHM}$ ) over a $400 \mu m$ diameter as shown in figure 9 . Such an aberration, leading to strong wavefront distortions after some round-trips in the laser resonator, should also be considered in the correction process.

\section{CONCLUSION}

In conclusion, the effects of thermally induced index variation on a probe beam injected in a $\mathrm{CW}$ diode end-pumped $\mathrm{YVO}_{4}$ laser have been characterized.

First of all, we have shown experimentally that under strong pumping power, the beam profile becomes multimodal without additional intra-cavity filtering. Thus, the phase retrieval cannot be achieved analyzing directly the laser beam. That is why thermal effect measurements were performed thanks to an external $\mathrm{TEM}_{00}$ probe beam. The emission wavelength of this probe $\left(\lambda_{p}=1079 \mathrm{~nm}\right)$ was chosen outside the $\mathrm{YVO}_{4}(\lambda=1064 \mathrm{~nm})$ gain bandwidth in order to avoid a 
change of the population inversion. The probe and laser beams have parallel polarizations and the recordings were obtained a few milliseconds after the laser shutdown. In this case, the measured thermal distortions are roughly those of the running laser.

After a round-trip in the laser medium the irradiance of the probe beam was recorded in the three wavefront planes: the image of the laser medium output plane, its Fourier transform plane and a Fresnel one.

We have developed a Gerchberg-Saxton-like numerical algorithm in order to reconstruct the phase profile of the distorted probe beam using irradiance measurements in three planes. The calculated phase pattern in the output plane of the laser crystal was analyzed by a Zernike polynomial expansion. Three major aberration terms were pointed out: defocusing (OPD of $0.7 \lambda_{p}$ FWHM) , spherical aberration (OPD of $0.3 \lambda_{p}$ FWHM) and first order astigmatism (OPD of $\pm 0.14 \lambda_{p}$ FWHM). However, the aberration composed by all higher order terms was found to be significant $\left(\lambda_{p} / 4\right.$ OPD FWHM). These distortions are dramatically enhanced in CW operation because of the high quality factor of the resonator and the low gain. Therefore, the whole aberration, including all the higher order terms, has to be taken into account in the phase distortion correction process.

[1] A. Brignon, L. Loiseau, C. Larat, J. P. Huignard, and J. P. Pocholle, Applied Physics B 19, 159 (1999).

[2] S. Mailis, J. Hendricks, D. P. Shepherd, A. C. Tropper, N. Moore, R. W. Eason, G. J. Crofts, M. Trew, and J. Damzen, Optics Letters 24, 972 (1999).

[3] U. Brauch, A. Giesen, M. Karszewski, C. Stewen, and A. Voss, Opt. Lett. 20, 713 (1995).

[4] W. A. Clarkson and D. C. Hanna, Resonator design considerations for efficient operation of solid-state lasers end-pumped by high-power diode-bars (Kluwer Academic Publishers, 1998), R. Kossowsky et al. ed.

[5] S. C. Tidwell, J. F. Seamens, M. S. Bowers, and A. Cousins, IEEE J. Quantum Electron. 28, 997 (1992).

[6] C. Roddier and F. Roddier, Applied Optics 32, 2992 (1993).

[7] R. W. Gerchberg and W. O. Saxton, Optik (Stuttgart) 35, 237 (1972).

[8] D. Brunson, Source files for determination of zernike polynmomial expansion (1997), ftp://ftp.mathworks.com/pub/contrib/v5/physics/zernikes/. 